\title{
Contaminación Urbana por Material Particulado y su Efecto sobre las Características Morfo-Anatómicas de Cuatro Especies Arbóreas de Santiago de Chile
}

\author{
Claudia Egas ${ }^{(1)}$, Paulette I. Naulin ${ }^{(2)}$ y Margarita Préndez ${ }^{(1) \star}$ \\ (1) Facultad de Ciencias Químicas y Farmacéuticas, Dpto. Química Orgánica y Fisicoquímica, Universidad de \\ Chile, Código postal 8380492, Santiago, Chile. (e-mail: mprendez@ciq.uchile.cl) \\ (2) Facultad de Ciencias Forestales y de la Conservación de la Naturaleza, Dpto. Silvicultura y Conservación \\ de la Naturaleza, Universidad de Chile, Código postal 8820808, Santiago, Chile.
}

*autor a quien debe ser dirigida la correspondencia

Recibido Nov. 22, 2017; Aceptado Feb. 5, 2018; Versión final Mar. 28, 2018, Publicado Ago. 2018

\begin{abstract}
Resumen
El objetivo de este trabajo es analizar el efecto de la contaminación sobre cuatro especies arbóreas urbanas sometidas a concentraciones diferentes de material particulado. El material particulado es el contaminante atmosférico que supera más frecuentemente la norma en las ciudades chilenas afectando la salud humana y ambiental. En este contexto, se estudiaron características morfo-anatómicas de las hojas de las especies Quillaja saponaria, Schinus molle, Olea europaea y Melia azedarach presentes en tres áreas de estudio expuestas a diferentes concentraciones del contaminante. El análisis estadístico mostró diferencias significativas en la densidad de estomas abaxial de Quillaja saponaria y Melia azedarach y en el ancho de estomas abaxial de Quillaja saponaria entre los sitios estudiados. Los resultados podrían evidenciar un efecto negativo del contaminante sobre el funcionamiento biológico de los árboles. Esto debe constituir una alerta para las autoridades respecto de los criterios necesarios para seleccionar las especies arbóreas para vegetar la ciudad de Santiago.
\end{abstract}

\section{Urban Pollution by Particulate Matter and its Effect on Morpho-Anatomical Characteristics of Four Tree Species in Santiago, Chile}

\begin{abstract}
The objective of this work is to analyze the effect of pollution on four urban tree species exposed to different concentrations of particulate matter. Particulate matter is the atmospheric pollutant more frequently exceeding the standards in Chilean cities, affecting human and environmental health. In this context, morpho-anatomical characteristics of the leaves of the species Quillaja saponaria, Schinus molle, Olea europaea and Melia azedarach present in three study areas with different particulate matter concentrations were studied. Statistical analysis showed significant differences in the abaxial stomatal density of Quillaja saponaria and Melia azedarach and in the abaxial stomatal width of Quillaja saponaria among the studied sites. The results could show a negative effect of the pollutant on the biological functioning of the trees. This should represent an alert for authorities about the criteria used for the selection of the species employed in the vegetation of the city of Santiago.
\end{abstract}

Keywords: morpho-anatomy of leaves; particulate matter; selection of tree species 


\section{INTRODUCCIÓN}

La contaminación atmosférica afecta a las ciudades por medio de gases y aerosoles atmosféricos o material particulado (MP). El MP es una mezcla compleja de partículas sólidas y líquidas de diferente origen, tamaño, forma y composición química (Grantz et al., 2003; Préndez, 1993) con impacto sobre la salud humana, los ecosistemas, la visibilidad y la infraestructura, y con consecuencias económicas y sociales. La fracción menor a $10 \mu \mathrm{m}$ de diámetro, MP10, contiene las llamadas fracción fina, MP2,5 y fracción gruesa, MP2,5-10 (partículas de 2,5 $\mu \mathrm{m}$ y entre 2,5 y $10 \mu \mathrm{m}$ de diámetro aerodinámico, respectivamente). La Organización Mundial de la Salud ha establecido una relación directa entre la exposición a altas concentraciones de partículas gruesas y finas y los niveles de mortalidad y morbilidad de la población estimando que dicha exposición puede causar 3,7 millones de muertes prematuras en todo el mundo (OMS, 2014). Si bien las partículas finas representan una menor proporción de la masa total del MP, su número es mayor y las partículas finas y ultrafinas (del orden de los nanómetros) presentan mayores efectos sobre la salud humana (Li et al., 2017). Químicamente, el MP contiene especies inorgánicas como sulfatos, nitratos y metales pesados ( $\mathrm{p}$. ej. $\mathrm{Fe}, \mathrm{Pb}, \mathrm{Cd}, \mathrm{Ni}, \mathrm{Cu}, \mathrm{Mo}$ y V), además de compuestos orgánicos entre los que se encuentran los hidrocarburos aromáticos policíclicos (Préndez, 1993). En Chile, la mayoría de las ciudades tienen problemas de calidad del aire; en particular Santiago, su capital, presenta concentraciones de MP2,5 y MP10 sobre la norma en el período otoño-invierno. Para el periodo entre abril y julio las concentraciones promedio de varios años para MP2,5 se sitúan entre 30$45 \mu \mathrm{g} / \mathrm{m}^{3} \mathrm{~N}$ y para MP10 entre $80-90 \mu \mathrm{g} / \mathrm{m}^{3} \mathrm{~N}$. Las normas primarias para material particulado MP10 son 150 $\mu \mathrm{g} / \mathrm{m}^{3} \mathrm{~N}$ promedio anual y $50 \mu \mathrm{g} / \mathrm{m}^{3} \mathrm{~N}$ en 24 horas. Para MP2,5 las normas son $50 \mu \mathrm{g} / \mathrm{m}^{3} \mathrm{~N}$ promedio anual y $20 \mu \mathrm{g} / \mathrm{m}^{3} \mathrm{~N}$ en 24 horas (MMA, 2018; Préndez et al., 2018).

Santiago se localiza en la cuenca del río Maipo; limita al este con la Cordillera de los Andes y al oeste con la Cordillera de la Costa. Al norte y al sur cierran la cuenca cadenas de cerros de 1500-2000 m; los episodios de contaminación por MP están influidos por las malas condiciones de ventilación que ello genera (Préndez et al., 2011). A su vez, el crecimiento de la población y la expansión urbana han sustituido tierras agrícolas y hábitats naturales (Romero et al., 2012), impactando negativamente la calidad del aire de la ciudad, disminuyendo la cubierta vegetal y aumentando las temperaturas (Krellenberg et al., 2013).

Diversos estudios disciplinarios realizados por agencias gubernamentales y universidades muestran los efectos negativos de la contaminación del aire. En Chile, pese a que se han establecido tácticas y estrategias por parte del Estado desde 1997, el problema vuelve a aparecer cada año. Algunas de las principales medidas se enfocaron en mejoras tecnológicas conducentes a la disminución de las emisiones de fuentes estacionarias y vehículos motorizados (Préndez et al., 2007; 2011). En algunos países sin embargo, la vegetación urbana se ha utilizado para mejorar la calidad del aire al influir en la dispersión de los contaminantes y/o al actuar como un filtro natural por intercepción de las partículas transportadas por el aire, mediante sus hojas, ramas y ramillas o por absorción de ellos a través de los estomas de las hojas (Janhäll, 2015). La fracción de partículas más gruesas suele eliminarse durante las precipitaciones (Przybysz et al., 2014); el depósito en la cera de la hoja se elimina más difícilmente (Dzierzanowski et al., 2011). La mayoría de las plantas tienen una gran superficie por unidad de volumen aumentando la probabilidad de depositación en un proceso que difiere sustancialmente con el tamaño de partícula y los elementos presentes en la superficie de la hoja (Janhäll, 2015). Los aerosoles atmosféricos capturados por las hojas de las plantas pueden ayudar también en la identificación de sus fuentes debido a su generación por diferentes procesos. Las partículas de más de $1 \mu \mathrm{m}$ y forma irregular provienen principalmente de fuentes naturales, mientras que las de menos de $1 \mu \mathrm{m}$, esféricas e irregulares, proceden principalmente de fuentes antropogénicas (Préndez, 1993). Las partículas de los tubos de escape de los vehículos diesel incluyen agregados de hollín (Akram et al., 2014) y partículas magnéticas (Muñoz et al., 2017).

La captura del MP es un servicio ecosistémico de la vegetación urbana especie-específico debido a las características fisiológicas, químicas y morfológicas de la planta, afectando significativamente su valor. La capacidad de retención depende principalmente de las características en la superficie de las hojas, tales como tricomas, rugosidad superficial, capa de cera epicuticular (Dzierzanowski et al., 2011), número de surcos y tricomas, pero no de la forma foliar y la venación de hoja ancha (Chen et al., 2017). Dicho conjunto de características podría usarse como indicador para la efectividad de la captura de MP2,5 por los árboles. Por su parte, el MP obstruye los estomas de las hojas (Gajbhiye et al., 2016), lo cual afectaría el intercambio gaseoso alterando la actividad fisiológica de la planta. Así, el conocimiento de las características de las especies es crítico, y plantar especies no apropiadas en lugares equivocados puede causar problemas no deseados (Willis y Petrokofsky, 2017).

En Santiago, se ha determinado mediante modelación, que la vegetación arbórea puede reducir contaminantes atmosféricos, tales como $\mathrm{O}_{3}, \mathrm{MP}, \mathrm{SO}_{2}, \mathrm{CO}$ y NOx (Escobedo et al., 2008). La investigación experimental del estudio de árboles en Santiago como filtradores de MP, por el método gravimétrico tradicional de Guerrero-Leiva et al. (2016) cuantificaron la cantidad de MP retenido por tres especies ornamentales mostrando que Nerium oleander L. retienen más material particulado que Pittosporum tobira (Thunb.) 
W.T.Aiton y Ligustrum lucidum W.T.Aiton; a su vez Muñoz et al. (2017) estimaron, mediante técnicas magnéticas, que las especies Platanus orientalis L. y Acer negundo L. capturan más MP que la especie Robinia pseudoacacia L. Dicho estudio realizado en dos comunas de la ciudad permitió asociar directamente el MP depositado sobre las hojas de las especies indicadas y el polvo depositado en las calles, con las emisiones de los tubos de escape de los vehículos, la principal fuente de aerosoles atmosféricos en Santiago (44\%, MMA, 2014). Sin embargo, se desconoce el efecto de dicho MP sobre la salud de las hojas de los árboles. El objetivo de este trabajo es precisamente estudiar el efecto de la contaminación por material particulado sobre algunas características morfo-anatómicas de cuatro especies arbóreas urbanas frecuentemente usadas en la forestación de la ciudad de Santiago de Chile.

\section{MATERIALES Y MÉTODO}

Se presentan tres subsecciones, correspondientes al lugar de estudio, las especies arbóreas a investigar y el procedimiento utilizado en el análisis de las hojas de cada especie. Es preciso mencionar que el MMA posee una red oficial de estaciones de monitoreo del aire que en la Región Metropolitana está dotada de 11 estaciones. En este trabajo uno de los sitios estudiados queda fuera del área de representatividad de una estación de monitoreo (radio de $2 \mathrm{~km}$ ).

\section{Sitio de muestreo}

La Figura 1 muestra las tres áreas de estudio ( $A, B$ y $C$ ) seleccionadas en las cuales se muestrearon las 4 especies arbóreas expuestas a concentraciones diferentes de material particulado: 1) Área A: Campus Sur de la Universidad de Chile (Av. Santa Rosa 1131), situada al sur-poniente de la ciudad es una zona urbana y agrícola en la dirección de la entrada matinal del aire fresco a la ciudad, con bajo flujo vehicular directo y escasa presencia de fuentes estacionarias (Seremi de Salud, 2018); 2) Área B: Campus Norte de la Universidad de Chile (Sergio Livingston 1007) correspondiente a una zona urbanizada con flujo vehicular medio-alto, a menos de 2 km al norte del centro cívico de la ciudad; 3) Área C correspondiente a una transecta de la Avenida Recoleta (números 3785 al 1624), arteria con alto flujo vehicular. El área B forma parte del área de representatividad de la Estación de Monitoreo Independencia. El área C está en el borde de dicha estación, pero las especies muestreadas tienen una exposición directa al flujo vehicular por lo que su exposición al contaminante es mayor. Además en esta zona se encuentra un mayor número de fuentes estacionarias (Seremi de Salud, 2018)
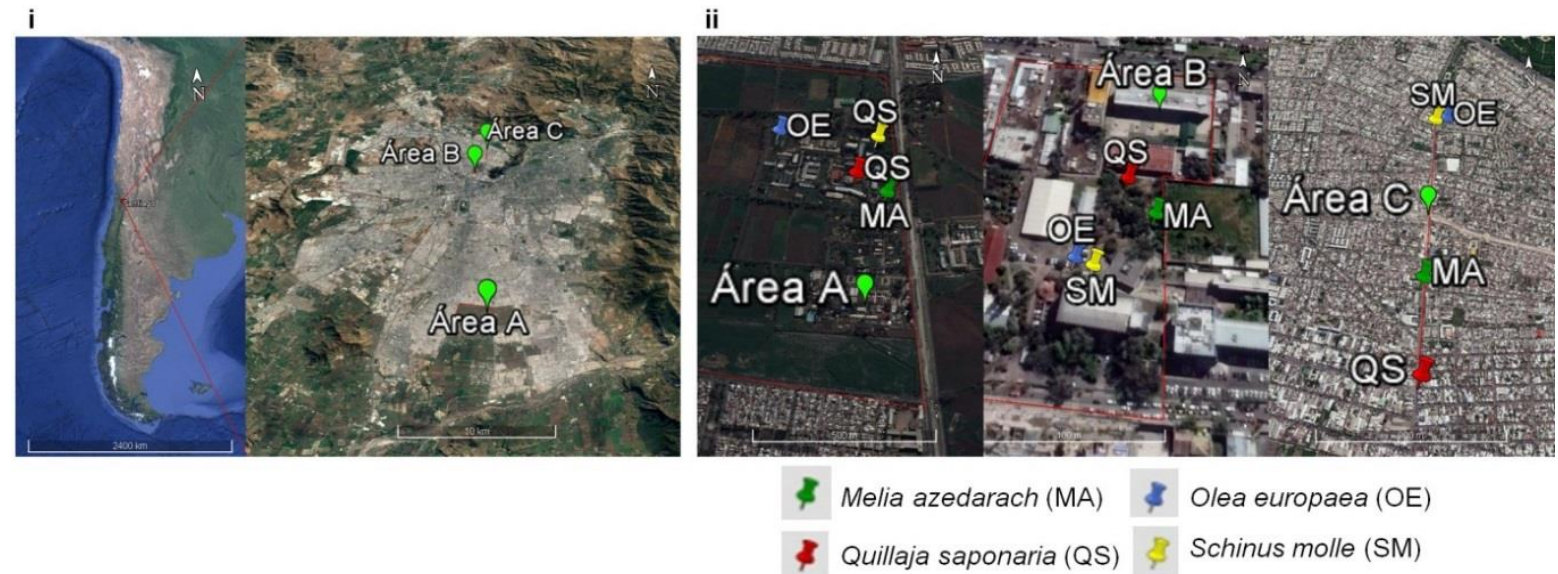

Fig. 1: (i) Áreas de colección del material vegetal de las cuatro especies arbóreas en Santiago de Chile. (ii) Distribución de las especies arbóreas en las áreas con diferentes niveles de contaminantes: Área A de bajo nivel de contaminación; Área B de medio-alto nivel de contaminación y Área $\mathrm{C}$ de alto nivel de contaminación por material particulado.

\section{Selección y colección de especies arbóreas}

Los árboles urbanos estudiados corresponden a las especies nativas: Quillaja saponaria Mol. (QS) y Schinus molle L. (SM) y a las especies introducidas: Melia azedarach L. (MA) y Olea europaea L. (OE), las que en conjunto representan el 7,3 \% de abundancia de especies arbóreas de la ciudad (3,5\%, 1,8\%, 1,4\% y 0,6\% respectivamente (Hernández y Villaseñor, 2018) y se seleccionaron por estar presentes en las tres áreas estudiadas. Las especies QS, SM y OE se caracterizan por ser perennes y la especie MA es caduca. Se colectaron cinco hojas (en el caso de QS y OE) y cinco foliolos (en el caso de SM y MA) para cada individuo de las especies en estudio en las áreas A, B y C, considerando sólo hojas frescas y completamente desarrolladas (maduras) y escogidas de secciones equivalentes del árbol (zona sur de la copa y del tercio inferior). El muestreo se realizó en diciembre de 2015. Tres muestras de las colectadas para cada especie se utilizaron en el análisis foliar. 


\section{Análisis foliar y estadístico}

Para cada muestra se utilizó el método de medición de áreas con escáner de luz HP Scanjet 3670 y el programa de procesamiento de imagen IMAGEJ (National Institute of Health, USA). Luego, se diafanizó el tercio central de cada muestra seleccionada con el fin de observar la epidermis adaxial (superior) y abaxial (inferior) y de caracterizar las variables morfo-anatómicas: largo estoma adaxial (LEADA), largo estoma abaxial (LEABA), ancho estoma adaxial (AEADA), ancho estoma abaxial (AEABA), densidad estoma adaxial (DEADA), densidad estoma abaxial (DEABA), área foliar (AF) y perímetro foliar (PF). Se utilizó una modificación del protocolo propuesto por Dizeo de Strimatter (1973), reemplazando el uso de alcohol por hipoclorito de sodio para mejor separación de las epidermis. Las preparaciones se fotografiaron con un microscopio de luz Carl Zeiss Axiostar, con una cámara digital Canon acoplada y se usó el programa IMAGEJ para medición del largo y ancho de los estomas. Se efectuaron 30 mediciones para cada epidermis en cada muestra con un aumento de 40X. La densidad estomática se determinó a partir del número de estomas en un área de $1 \mathrm{~mm}^{2}$ para cada epidermis en cada muestra con un aumento de 10X, según el método propuesto por Dunlap y Steller (2001). La superficie de una hoja de cada una de las cuatro especies en las tres áreas (A, B, C) se metalizó y analizó con el Microscopio Electrónico de Barrido Ambiental (ESEM ME10 Zeiss) con un aumento de 2500X y una sonda electrónica asociada (EDX) al software AZtec (Oxford Instruments, UK) para el análisis elemental. Se utilizó la prueba de Kruskal-Wallis para evaluar las diferencias entre las áreas de estudio para cada una de las variables morfo-anatómicas estudiadas en las cuatro especies arbóreas.

\section{RESULTADOS Y DISCUSIÓN}

Los resultados indican que: 1) las hojas de QS y SM presentan estomas en ambas epidermis, esto es, son anfiestomáticas (Valenzuela, 2014; Ferreira Pires et al., 2014, respectivamente). OE, descrita por Bongi et al., (1987) y MA sólo presentan estomas en la epidermis abaxial, son hipoestomáticas. Las hojas de las cuatro especies presentan modificaciones epidermales (tricomas); en particular, las hojas de OE presentan tricomas en forma de sombrillas en ambas epidermis (escamas), con mayor abundancia en la epidermis abaxial (como se muestra en las Figuras 2 y 3 ). La Tabla 1 muestra los valores promedio, con sus respectivas desviaciones estándar, de las variables morfo-anatómicas: largo, ancho y densidad de estomas en ambas epidermis de las cuatro especies arbóreas estudiadas en las tres áreas de la ciudad. En la Tabla, QS: Quillaja saponaria, SM: Schinus molle, OE: Olea europaea, MA: Melia azedarach, largo estoma adaxial (LEADA), largo estoma abaxial (LEABA), ancho estoma adaxial (AEADA), ancho estoma abaxial (AEABA), densidad estoma adaxial (DEADA), densidad estoma abaxial (DEABA), área foliar (AF), perímetro foliar (PF), n/a: no aplica.

Tabla 1: Valores promedio de variables morfo-anatómicas con sus respectivas desviaciones estándar, de cuatro especies arbóreas en las tres áreas urbanas (A, B y C).

\begin{tabular}{|c|c|c|c|c|c|c|c|c|c|}
\hline 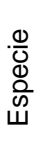 & $\stackrel{\mathbb{N}}{\stackrel{\mathbb{2}}{<}}$ & 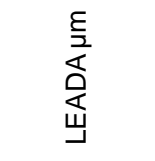 & 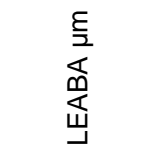 & 冢 & 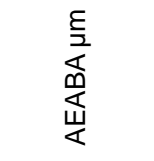 & 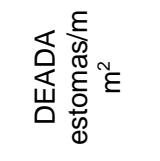 & 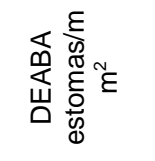 & 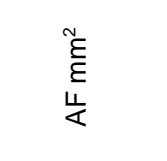 & $\begin{array}{l}\underline{\xi} \\
\underline{\varepsilon} \\
\stackrel{u}{\alpha}\end{array}$ \\
\hline \multirow{3}{*}{$\mathscr{O}$} & $\begin{array}{c}\text { Área } \\
\text { A }\end{array}$ & $\begin{array}{c}12,05 \pm 0,31 \\
4\end{array}$ & $\begin{array}{c}15,07 \pm 0,38 \\
6\end{array}$ & $\begin{array}{c}5,627 \pm 0,06 \\
0\end{array}$ & $\begin{array}{c}7,381 \pm 0,13 \\
4\end{array}$ & $\begin{array}{c}6,667 \pm 3,30 \\
0\end{array}$ & $\begin{array}{c}152,0 \pm 4,32 \\
0\end{array}$ & $1148 \pm 67,87$ & $\begin{array}{c}142,7 \pm 4,49 \\
1\end{array}$ \\
\hline & $\begin{array}{c}\text { Área } \\
\text { B }\end{array}$ & $\begin{array}{c}12,77 \pm 0,85 \\
2\end{array}$ & $\begin{array}{c}13,24 \pm 0,69 \\
8\end{array}$ & $\begin{array}{c}5,439 \pm 0,20 \\
5\end{array}$ & $\begin{array}{c}6,304 \pm 0,20 \\
1\end{array}$ & $\begin{array}{c}30,33 \pm 14,0 \\
1\end{array}$ & $\begin{array}{c}215,3 \pm 10,4 \\
0\end{array}$ & $\begin{array}{c}665,4 \pm 58,1 \\
1\end{array}$ & $\begin{array}{c}112,09 \pm 2,5 \\
2\end{array}$ \\
\hline & $\begin{array}{c}\text { Área } \\
\text { C }\end{array}$ & $\begin{array}{c}13,69 \pm 1,26 \\
8\end{array}$ & $\begin{array}{c}13,75 \pm 0,13 \\
2 \\
\end{array}$ & $\begin{array}{c}6,394 \pm 0,90 \\
2 \\
\end{array}$ & $\begin{array}{c}7,045 \pm 0,31 \\
2 \\
\end{array}$ & $\begin{array}{c}6,000 \pm 7,78 \\
9\end{array}$ & $\begin{array}{c}243,3 \pm 21,4 \\
5 \\
\end{array}$ & $\begin{array}{c}598,5 \pm 10,7 \\
1\end{array}$ & $\begin{array}{c}114,3 \pm 11,5 \\
9\end{array}$ \\
\hline \multirow{3}{*}{$\sum_{\infty}$} & $\begin{array}{c}\text { Área } \\
\mathrm{A}\end{array}$ & $\begin{array}{c}17,50 \pm 0,87 \\
4 \\
\end{array}$ & $\begin{array}{c}17,26 \pm 0,21 \\
2 \\
\end{array}$ & $\begin{array}{c}9,409 \pm 1,20 \\
0 \\
\end{array}$ & $\begin{array}{c}10,17 \pm 0,56 \\
7 \\
\end{array}$ & $\begin{array}{c}76,00 \pm 6,97 \\
6 \\
\end{array}$ & $\begin{array}{c}190,0 \pm 18,8 \\
3 \\
\end{array}$ & $\begin{array}{c}99,84 \pm 5,55 \\
6 \\
\end{array}$ & $\begin{array}{c}61,14 \pm 5,44 \\
4 \\
\end{array}$ \\
\hline & $\begin{array}{c}\text { Área } \\
\text { B }\end{array}$ & $\begin{array}{c}18,09 \pm 0,52 \\
9\end{array}$ & $\begin{array}{c}17,99 \pm 0,51 \\
1\end{array}$ & $\begin{array}{c}9,780 \pm 0,40 \\
0\end{array}$ & $\begin{array}{c}10,20 \pm 0,08 \\
5\end{array}$ & $\begin{array}{c}78,33 \pm 7,71 \\
7\end{array}$ & $\begin{array}{c}125,3 \pm 8,73 \\
1\end{array}$ & $\begin{array}{c}118,6 \pm 7,17 \\
8\end{array}$ & $\begin{array}{c}68,79 \pm 4,58 \\
2\end{array}$ \\
\hline & $\begin{array}{c}\text { Área } \\
\text { C }\end{array}$ & $\begin{array}{c}17,51 \pm 1,03 \\
5\end{array}$ & $\begin{array}{c}16,88 \pm 1,65 \\
7\end{array}$ & $\begin{array}{c}9,274 \pm 1,07 \\
2\end{array}$ & $\begin{array}{c}8,593 \pm 0,86 \\
9\end{array}$ & $\begin{array}{c}96,33 \pm 10,8 \\
7\end{array}$ & $\begin{array}{c}123,7 \pm 25,3 \\
2\end{array}$ & $\begin{array}{c}95,33 \pm 15,7 \\
1\end{array}$ & $\begin{array}{c}51,76 \pm 4,83 \\
7\end{array}$ \\
\hline \multirow{3}{*}{ О } & $\begin{array}{c}\text { Área } \\
\text { A }\end{array}$ & $\mathrm{n} / \mathrm{a}$ & $\begin{array}{c}15,22 \pm 1,44 \\
0 \\
\end{array}$ & $\mathrm{n} / \mathrm{a}$ & $\begin{array}{c}7,597 \pm 0,78 \\
7 \\
\end{array}$ & 0,0 & $\begin{array}{c}300,0 \pm 39,2 \\
5 \\
\end{array}$ & $\begin{array}{c}590,9 \pm 72,9 \\
5 \\
\end{array}$ & $\begin{array}{c}153,1 \pm 6,32 \\
5 \\
\end{array}$ \\
\hline & $\begin{array}{c}\text { Área } \\
\text { B }\end{array}$ & $\mathrm{n} / \mathrm{a}$ & $\begin{array}{c}15,68 \pm 0,59 \\
1\end{array}$ & $\mathrm{n} / \mathrm{a}$ & $\begin{array}{c}6,589 \pm 0,53 \\
9\end{array}$ & 0,0 & $\begin{array}{c}345,7 \pm 8,99 \\
4\end{array}$ & $\begin{array}{c}630,5 \pm 103 \\
5\end{array}$ & $\begin{array}{c}166,4 \pm 20,1 \\
0\end{array}$ \\
\hline & $\begin{array}{c}\text { Área } \\
\text { C }\end{array}$ & $\mathrm{n} / \mathrm{a}$ & $\begin{array}{c}15,91 \pm 0,59 \\
8 \\
\end{array}$ & $n / a$ & $\begin{array}{c}7,074 \pm 0,27 \\
4\end{array}$ & 0,0 & $\begin{array}{c}380,3 \pm 48,4 \\
7\end{array}$ & $\begin{array}{c}346,1 \pm 20,6 \\
5\end{array}$ & $\begin{array}{c}105,3 \pm 0,74 \\
5\end{array}$ \\
\hline \multirow{3}{*}{$\sum$} & $\begin{array}{c}\text { Área } \\
\text { A }\end{array}$ & $\mathrm{n} / \mathrm{a}$ & $\begin{array}{c}13,30 \pm 0,18 \\
4\end{array}$ & $\mathrm{n} / \mathrm{a}$ & $\begin{array}{c}6,072 \pm 0,25 \\
6\end{array}$ & 0,0 & $\begin{array}{c}274,3 \pm 6,94 \\
4\end{array}$ & $\begin{array}{c}456,5 \pm 32,7 \\
9\end{array}$ & $\begin{array}{c}117,2 \pm 4,86 \\
2\end{array}$ \\
\hline & $\begin{array}{c}\text { Área } \\
\text { B }\end{array}$ & $\mathrm{n} / \mathrm{a}$ & $\begin{array}{c}13,14 \pm 0,44 \\
6 \\
\end{array}$ & $\mathrm{n} / \mathrm{a}$ & $\begin{array}{c}5,848 \pm 0,50 \\
5 \\
\end{array}$ & 0,0 & $\begin{array}{c}288,7 \pm 23,5 \\
7\end{array}$ & $\begin{array}{c}485,2 \pm 18,0 \\
1\end{array}$ & $\begin{array}{c}130,9 \pm 13,9 \\
2\end{array}$ \\
\hline & $\begin{array}{c}\text { Área } \\
\text { C }\end{array}$ & $\mathrm{n} / \mathrm{a}$ & $\begin{array}{c}14,81 \pm 0,14 \\
2 \\
\end{array}$ & $n / a$ & $\begin{array}{c}6,379 \pm 0,41 \\
1 \\
\end{array}$ & 0,0 & $\begin{array}{c}232,0 \pm 14,1 \\
7 \\
\end{array}$ & $\begin{array}{c}715,6 \pm 28,0 \\
1 \\
\end{array}$ & $\begin{array}{c}143,9 \pm 3,46 \\
5 \\
\end{array}$ \\
\hline
\end{tabular}


Se observa que el largo y ancho de estomas de la epidermis adaxial y abaxial de QS y abaxial de MA y OE son menores en relación a ambas epidermis de SM. Por otra parte, la densidad de estomas en la epidermis adaxial es menor en QS que en SM y la densidad de estomas en la epidermis abaxial es menor en las hojas de SM que en las de OE.

Los valores de densidad de estomas obtenidos para el área $C$ en este trabajo se compararon con los resultados de Ferreira Pires et al. (2014) para SM. Para la densidad de estomas adaxial nuestro resultado $\left(96,3 \pm 10,9\right.$ estomas $\left./ \mathrm{mm}^{2}\right)$ es comparable al valor promedio de 97,6 estomas $/ \mathrm{mm}^{2}$ obtenido por dichos autores a $1,55 \mathrm{~m}$; para la densidad de estomas abaxial nuestros resultados $\left(124 \pm 25,3\right.$ estomas $\left./ \mathrm{mm}^{2}\right)$ difieren de los 101,9 estomas $/ \mathrm{mm}^{2}$ informados por dichos autores. Por otra parte, los individuos de QS en las tres áreas de estudio presentan mayor superficie foliar $\left(804 \pm 205 \mathrm{~mm}^{2}\right)$, desplegando sus hojas en mayor medida que las de QS de la Reserva Nacional Rio Clarillo $\left(412 \mathrm{~mm}^{2}\right)$ (Valenzuela, 2014). Esta Reserva es un área natural libre de contaminación ubicada en la comuna de Pirque, situada en la precordillera, al sureste de Santiago, a una altitud entre 850 y 3.500 msnm.

La prueba de Krustal-Wallis aplicada a las variables morfo-anatómicas de las cuatro especies arbóreas en las tres áreas: A, B y C (valores en Tabla 1), entregó diferencias significativas en la densidad de estomas y en el ancho de estomas abaxial de QS $(\mathrm{H}: 6,49 \mathrm{p}<0,05)$. La densidad de estomas abaxial mostró diferencias significativas entre los sitios estudiados para MA ( $\mathrm{H}: 5,96 \mathrm{p}<0,05)$. Bruno et al. (2007) y Arriaga et al. (2014) encontraron diferencias significativas entre áreas con diferentes niveles de contaminación y variables morfoanatómicas para las especies Ligustrum lucidum y Fraxinus pennsylvanica y Ficus benjamina, respectivamente. Esta es una información a considerar por cuanto dichas especies se utilizan también en la vegetación urbana de Santiago. Con un menor número de estomas y de menor tamaño, la planta puede defenderse mejor de la absorción de contaminantes, pero también puede aumentar su obstrucción y reducir su fotosíntesis y por lo tanto su crecimiento (Pourkhabbaz et al., 2010).

Las Figuras 2 y 3 muestran la superficie de las epidermis adaxial y abaxial de las cuatro especies arbóreas en las tres áreas de estudio (A, B y C) y la diversidad de partículas en cuanto a tamaños y formas en ambas epidermis. Se observan menos partículas gruesas en el área $A$, que en el área $C$. La especie SM muestra mayores rugosidades en el sitio de mayor contaminación $(C)$ que en el de menor contaminación $(A)$. Sistema de defensa? Por otra parte, MA es la especie con mayor rugosidad en su superficie y son claramente visibles los característicos tricomas de OE que protegen los estomas del impacto directo del MP.

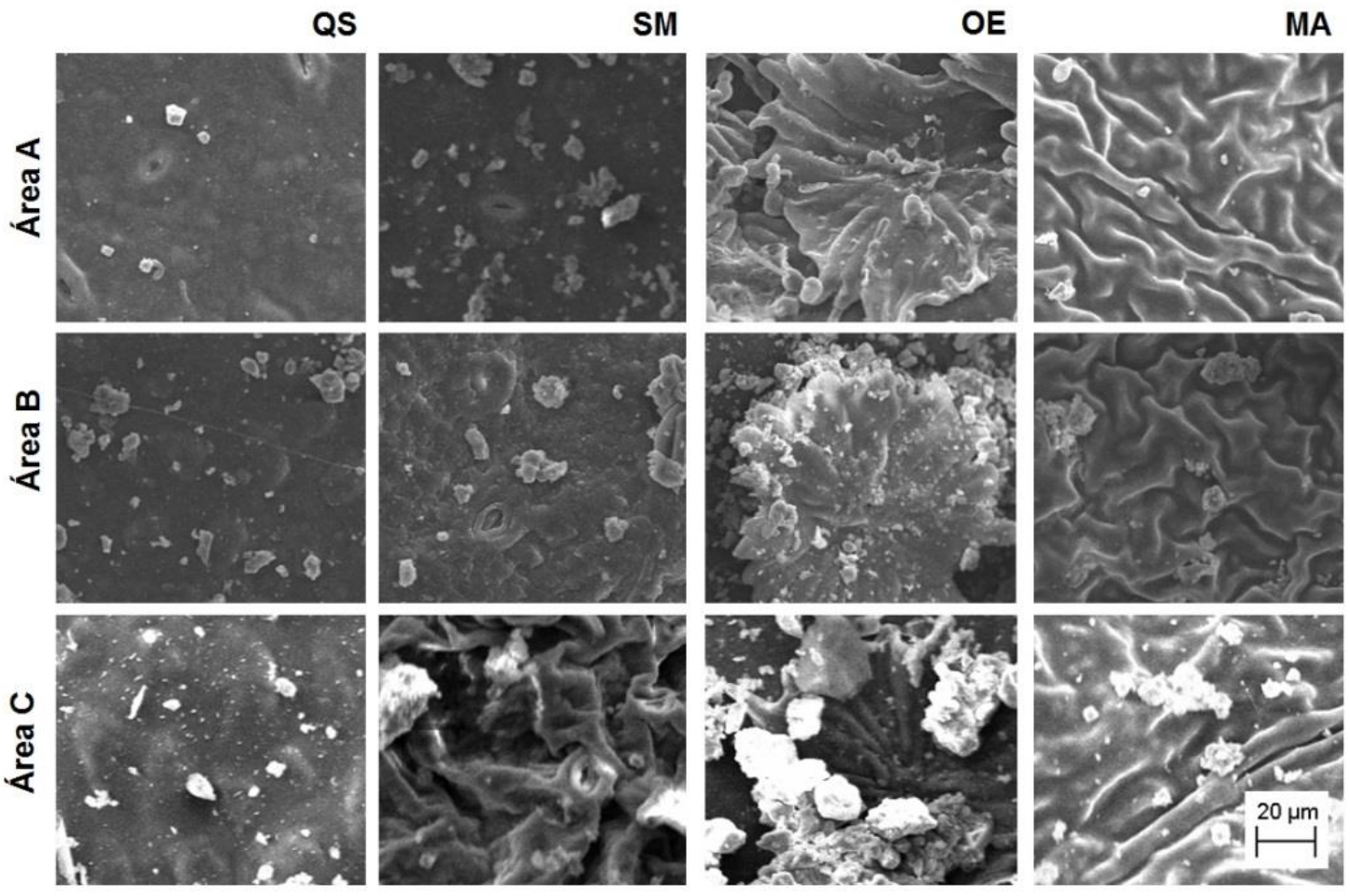

Fig. 2: Micrografías de las epidermis adaxial de QS: Quillaja saponaria, SM: Schinus molle, OE: Olea europaea, MA: Melia azedarach en las tres áreas de estudio: A, B y C. Aumento 2500X.

La Figura 4 muestra la presencia de material particulado depositado en la superficie de las hojas muy próximo o sobre los poros estomáticos en todas las especies en el área C. 
La presencia de MP sobre la epidermis y principalmente sobre los poros estomáticos bloquea la abertura estomática (Gajbhiye et al., 2016), de modo que la macro y micro-morfología de una superficie foliar se podría emplear para evaluar el impacto de la contaminación por MP, en acuerdo con Sawidis et al. (2012).

El análisis elemental de las micrografías permitió observar y analizar las partículas ultrafinas $(<0,1 \mu \mathrm{m})$ y brillantes depositadas en la superficie foliar. La Figura 5 muestra los espectros elementales de algunas partículas seleccionadas con un alto contenido en Fe. Los vehículos diésel por su combustible y temperatura de combustión, emiten material particulado esférico con partículas magnéticas correspondientes a magnetita, $\mathrm{Fe}_{3} \mathrm{O}_{4}$ (Matzka y Maher, 1999). Partículas ricas en Fe no esféricas pueden generarse por desgaste de los neumáticos y frenos, abrasión de partes de diferentes vehículos y pavimentos de carreteras (Matzka y Maher, 1999). La presencia de partículas magnéticas asociadas a Fe permitió así la identificación de MP proveniente de fuentes vehiculares sobre las hojas de los árboles estudiados.

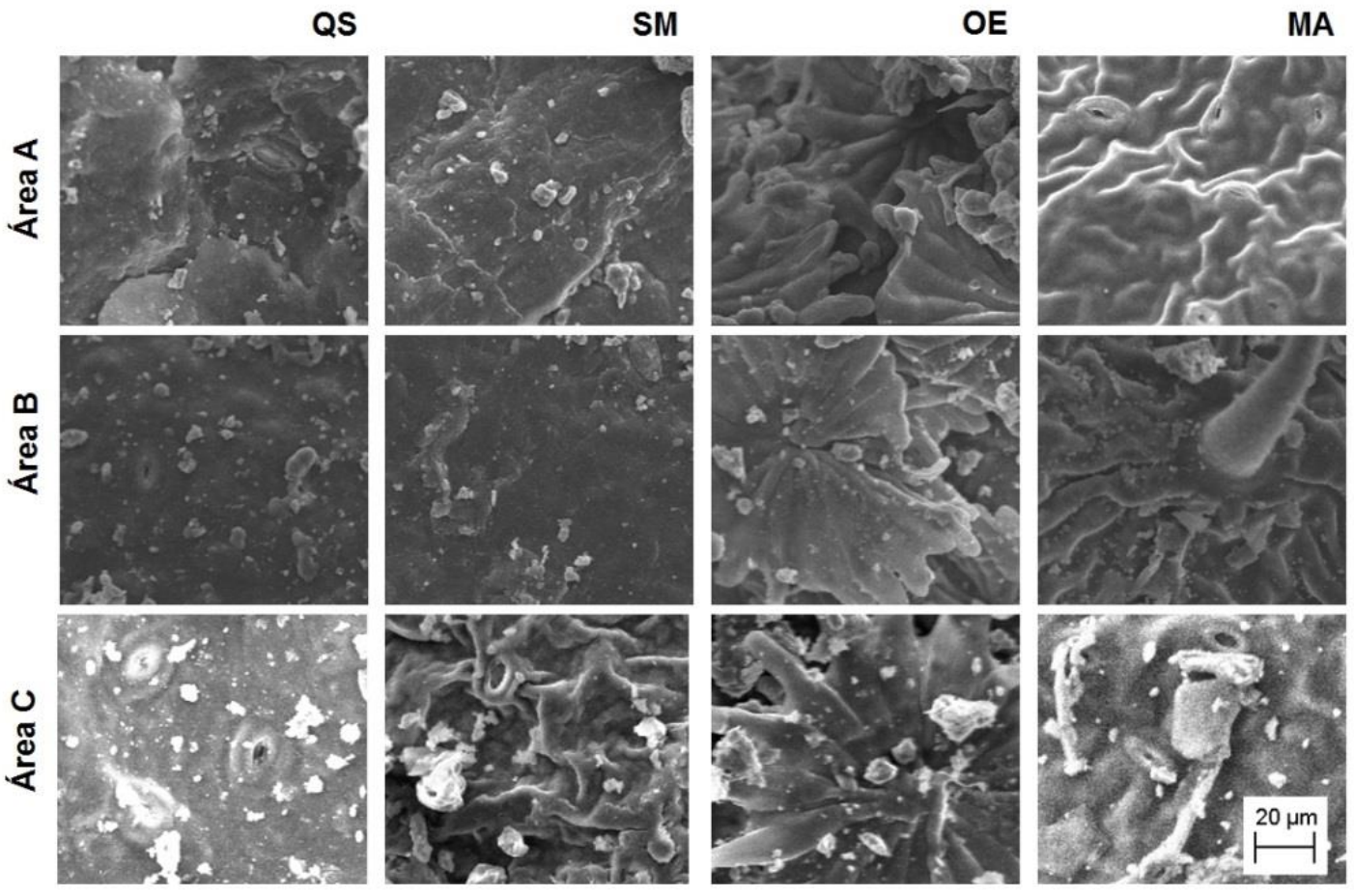

Fig. 3: Micrografías de las epidermis abaxial de QS: Quillaja saponaria, SM: Schinus molle, OE: Olea europaea, MA: Melia azedarach en las tres áreas de estudio. Aumento 2500X.
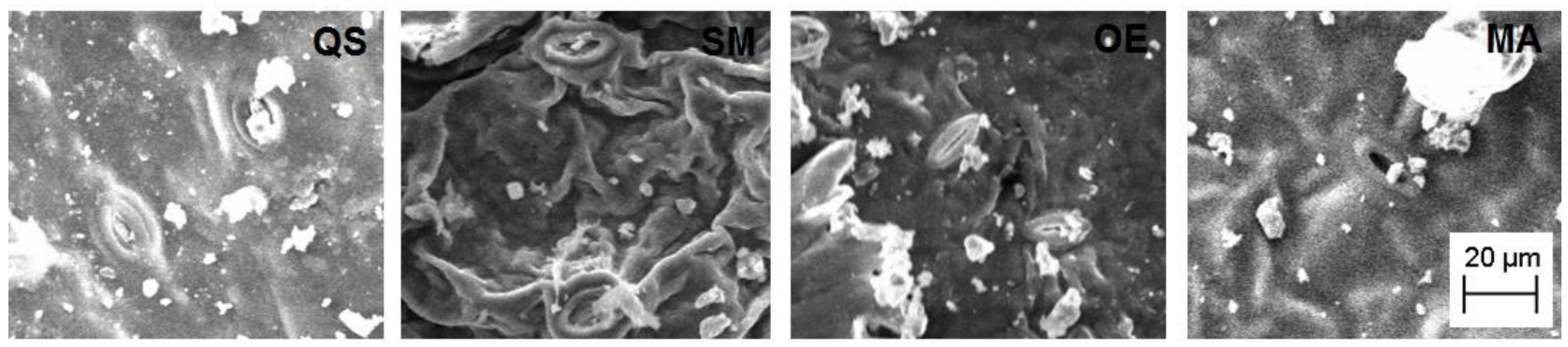

Fig. 4: Micrografías de estomas de epidermis abaxial de QS: Quillaja saponaria, SM: Schinus molle, OE: Olea euroapea, MA: Melia azedarach en el área $\mathrm{C}$ de alto nivel de contaminación por material particulado. Aumento 2500X.

De acuerdo con Singh et al. (2017) existe un efecto de la concentración de material particulado sobre la actividad fisiológica de las hojas, lo que también se observa en el presente trabajo. Esto releva la importancia en la selección de especies para la forestación urbana. Por otra parte, en el caso de Santiago con concentraciones ambientales de MP mayores en el período otoño-invierno resulta importante que las especies seleccionadas posean hojas en dicha época. En este trabajo se evidenciaron efectos sobre cuatro especies de las cuales tres de ellas, los árboles nativos $S$. molle y $Q$. saponaria y el árbol introducido 0 . europea, son especies siempre verdes y por lo tanto capturan y/o interceptan dicho contaminante durante todo el año. Sin embargo, $O$. europaea emite granos de polen con capacidad de generar alergia y afectar la salud humana. 
i

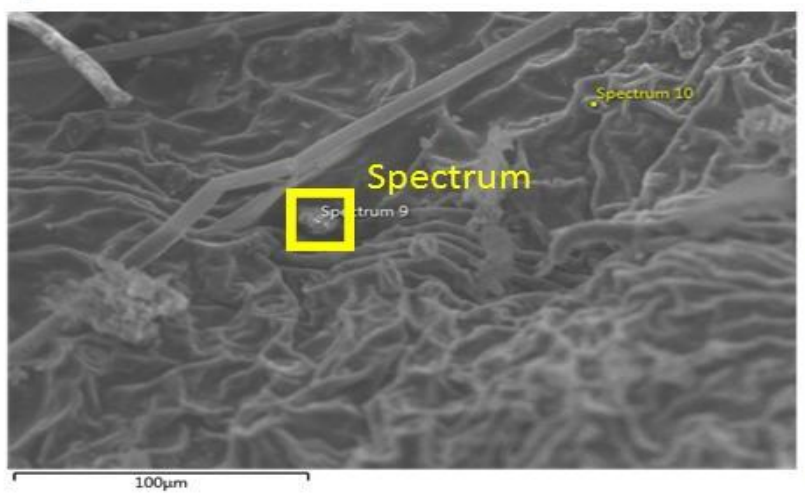

ii

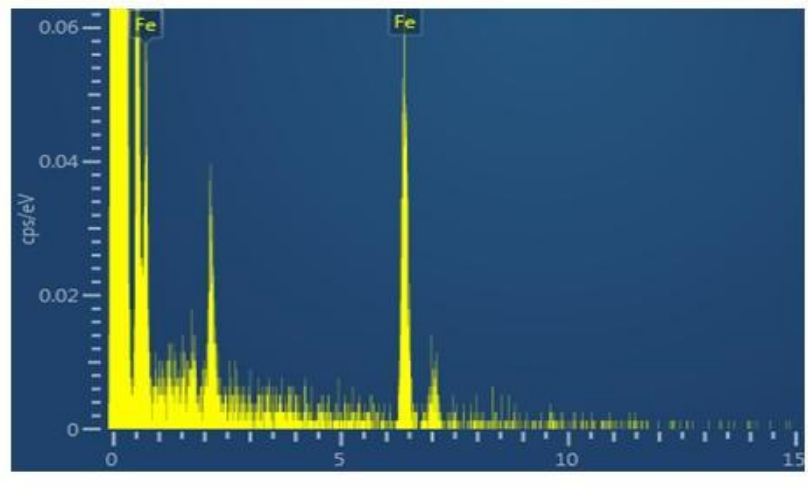

Fig. 5: (i) Micrografía con partícula depositada en epidermis abaxial de hoja de Schinus molle. (ii) espectro del análisis de SEM/EDX sobre la partícula utilizando software AZtec.

\section{CONCLUSIONES}

Los resultados morfo-anatómicos para Schinus molle, Quillaja saponaria, Olea europaea y Melia azedarach que se muestran en este trabajo son los primeros antecedentes del efecto que produce la contaminación por material particulado sobre las hojas de los árboles urbanos en Santiago.

Las cuatro especies estudiadas presentan características en la superficie física de sus epidermis capaces de retener partículas magnéticas de fuentes móviles y partículas de diversas formas y tamaños de interés para evaluar la extensión y el impacto de la contaminación por material particulado.

Los diferentes grados de contaminación del aire afectan la densidad y el ancho de estomas de las hojas de Quillaja saponaria y Melia azedarach por lo que sería esperable que altas concentraciones de material particulado afectaran el funcionamiento biológico de los árboles.

La captura de contaminantes resulta más eficiente si se selecciona la especie arbórea basada en las características morfo-anatómicas adecuadas ya que pueden maximizar la captura de contaminantes atmosféricos, mejorar la gestión del arbolado y la calidad de aire, e incluso potenciarse otros servicios ecosistémicos.

La selección de especies para la forestación urbana debe ser cuidadosamente estudiada, puesto que diferentes características de ellas son totalmente especie específica.

\section{AGRADECIMIENTOS}

Al proyecto URC-026/17 por el financiamiento para la publicación de este artículo.

\section{REFERENCIAS}

Akram, W., M. Madhuku y otros cuatro autores, Morphology, microstructure and chemical composition of single inhalable particles in Shanghai, China, Environ. Monit. Assess, 186(12), 8587-8598 (2014)

Arriaga, M., M. Stampacchio y otros tres autores, Use of epidermal characters as bioindicatorsof environmental pollution, Multequina, 23, 41-53 (2014)

Bruno, G., L. Stiefkens y otros cuatro autores, Efecto de la contaminación ambiental en la anatomía de la hoja de Ligustrum lucidum (Oleaceae), Boletín de la Sociedad Argentina de Botánica, 42(3-4), 231-236 (2007)

Bongi, G., M. Mencuccini y G. Fontanazza, Photosynthesis of olive leaves: effect of light flux density, leaf age, temperature, peltates, and $\mathrm{H}_{2} \mathrm{O}$ vapor pressure deficit on gas exchange, J. amer. Soc. hort. Sci., 112, 143-148 (1987)

Chen, L., C. Liu y otros cuatro autores, Variation in Tree Species Ability to Capture and Retain Airborne Fine Particulate Matter (PM2.5), Scientific Reports, 7, 3206 (2017)

Dizeo de Strittmatter, C., Nueva técnica de diafanización, Bol. Soc. Arg. Bot., 15(1), 126-129 (1973)

Dunlap, J. y R. Stettler, Variation in leaf epidermal and stomatal traits of Populus trichocarpa from two transects across the Washington Cascades, Canadian Journal of Botany, 79(5), 528-535 (2001)

Dzierzanowski, K., R. Popek y otros tres autores, Deposition of particulate matter of different size fractions on leaf surfaces and in waxes of urban forest species, Int. J. Phytoremediation, 13, 1037-1046 (2011)

Escobedo, F.J., J.E. Wagner y otros cuatro autores, Analyzing the cost-effectiveness of Santiago, Chile's policy of using urban forests to improve air quality, Journal of Environmental Management, 86, 148-157 (2008) 
Ferreira Pires, M.F., M.P. Pereira y otros tres autores, Micromorfometria foliar de Schinus molle L. (Anarcadiaceae) em diferentes alturas na copa, CERNE, 21(1), 17-25 (2014)

Gajbhiye, T., S. Kumar y otros tres autores, Science of the Total Environment Airborne foliar transfer of PM bound heavy metals in Cassia siamea: A less common route of heavy metal accumulation, Science of the Total Environment, 573,123$130(2016)$

Grantz, DA., J.H. Garner y D.W. Johnson, Ecological effects of particulate matter, Environment International, 29(2), 213239 (2003)

Guerrero-Leiva, N., S.A. Castro y otros dos autores, Retention of Atmospheric Particulate by Three Woody Ornamental Species in Santiago, Chile, Water, Air \& Soil Pollution, 227(12), 435 (2016)

Hernández, H.J. y N. Villaseñor, Twelve-year change in tree diversity and spatial segregation in the Mediterranean city of Santiago, Chile, Urban Forestry \& Urban Greening, 29, 10-18 (2018)

Janhäll, S., Review on urban vegetation and particle air pollution-Deposition and dispersion, Atmospheric Environment, $105,130-137$ (2015)

Krellenberg, K., A. Müller y A. Schwarz, Flood and heat hazards in the Metropolitan Region of Santiago de Chile and the socio- economics of exposure, Applied Geography, 38, 86-95 (2013)

$\mathrm{Li}, \mathrm{H} ., \mathrm{H}$. Ji y otros siete autores, Distribution of heavy metals and metalloids in bulk and particle size fractions of soils from coal-mine brownfield and implications on human health, Chemosphere, 172, 505-515 (2017)

Matzka, J. y B.A. Maher, Magnetic biomonitoring of roadside tree leaves: identification of spatial and temporal variations in vehicle-derived particulates, Atmospheric Environment, 33, 4565-4569 (1999)

MMA, Ministerio de Medio Ambiente: Planes de Descontaminación Atmosférica Estrategia 2014-2018 (2014)

MMA, Ministerio de Medio Ambiente: Actos y documentos publicados en el Diario Oficial (2018)

Muñoz, D., B. Aguilar y otros dos autores, Environmental studies in two communes of Santiago de Chile by the analysis of magnetic properties of particulate matter deposited on leaves of roadside trees, Atmospheric Environment, 152, 617627 (2017)

OMS, Organización Mundial de la Salud: Ambient (outdoor) air quality and health (2017)

Pourkhabbaz, A., N. Rastin y otros tres autores, Influence of environmental pollution on leaf properties of urban plane trees, Platanus orientalis L, Bulletin of Environmental Contamination and Toxicology, 85, 251-255 (2010)

Préndez, M., M. Araya y otros cinco autores, Urban trees and its relationships with air pollution by particulate matter and ozone in Santiago de Chile, in Urban Climate in Latin-American Cities by C. Henriquez \& H. Romero, Springer, en prensa (2018)

Préndez, M., G. Alvarado e I. Serey, Some Guidelines to Improve Air Quality Management in Santiago, Chile: from Commune to Basin level, in Air Quality Monitoring, Assessment and Management by N. Mazzeo, InTech (2011)

Préndez, M., R. Corvalán y M. Cisternas, Estudio preliminar del material particulado de fuentes estacionarias: Aplicación al sistema de compensación de emisiones en la Región Metropolitana, Chile, Información Tecnológica, 18(2), 93-103 (2007)

Préndez, M., Características de los Contaminantes Atmosféricos, in Contaminación Atmosférica de Santiago: Estado Actual y Soluciones by $\mathrm{H}$. Sandoval, Universidad de Chile y Comisión Especial de Descontaminación de la Región Metropolitana, Santiago, Chile (1993)

Przybysz, A., A. Sæbø y otros dos autores, Accumulation of particulate matter and trace elements on vegetation as affected by pollution level, rainfall and the passage of time, Science of the Total Environment, 481, 360-369 (2014)

Romero, H., A. Vásquez y otros cuatro autores, Assessing urban environmental segregation (UES). The case of Santiago de Chile, Ecological Indicators, 23, 76-87 (2012)

Sawidis, T., P. Krystallidis y otros dos autores, A study of air pollution with heavy metals in Athens city and Attica basin using evergreen trees as biological indicators, Biological trace element research, 148(3), 396-408 (2012)

Seremi de Salud, Listado de fuentes fijas que paralizan en contingencias ambientales (2018)

Singh, H., R. Sharma y otros seis autores, Physiological functioning of Lagerstroemia speciosa L. under heavy roadside traffic: an approach to screen potential species for abatement of urban air pollution, 3 Biotech, 7(1), 61 (2017)

Valenzuela, G., Caracterización de variables epidérmicas para la determinación del origen botánico de tierra de hojas de litre, en la Reserva Nacional Río Clarillo (Región Metropolitana), Memoria para optar al Título Profesional de Ingeniero Forestal, Facultad de Ciencias Forestales y de la Conservación de la Naturaleza. Universidad de Chile, Chile (2014)

Willis, K. J. y G. Petrokofsky, The natural capital of city trees, Science, 356(6336), 374-376 (2017) 